\title{
O PET-Saúde em consonância com as Diretrizes Curriculares Nacionais de Odontologia no desenvolvimento de competências profissionais: relato de experiência
}

\author{
Beatriz Sartori da Silva*, Pablo Guilherme Caldarelli** \\ * Acadêmica da $5^{\text {a }}$ série do curso de Odontologia da Universidade Estadual de \\ Londrina- UEL. Bolsista do Programa PET-SAÚDE. \\ ** Doutorando em Odontologia (Cariologia) pela Faculdade de Odontologia de \\ Piracicaba- FOP- UNICAMP.
}

\section{RESUMO}

As Diretrizes Curriculares Nacionais de Odontologia (DCNs) definem os objetivos do curso assim como os princípios, fundamentos, condições e procedimentos da formação de cirurgiões-dentistas e devem ter aplicação nacional sendo utilizadas como eixo orientador na elaboração dos currículos implementados em instituições de ensino superior (IES). A Universidade Estadual de Londrina (UEL) buscando atingir o patamar exigido pelas DCNs embasou asua matriz curricular em módulos integrados e em consonância com os mesmos aderiu ao Programa de Educação pelo trabalho para Saúde (PET-Saúde). O presente trabalho objetivou relatar as experiências dos autores deste artigo, que vivenciaram ativamente o PET-Saúde da UEL como facilitador em suas formações profissionais. $\mathrm{O}$ relato foi organizado em tópicos referentes às competências profissionais exigidas pelas DCNs do curso de Odontologia que foram desenvolvidas durante a experiência. Conclui-se que tanto as atividades desenvolvidas pelo PET-Saúde quanto as preconizadas pela matriz curricular do curso de Odontologia da Universidade Estadual de Londrina priorizam formar profissionais mais decisivos e desenvoltos, com conhecimentos e atividades que os tornem competentes para atuar no Sistema Único de Saúde (SUS), com amadurecimento profissional e elevada formação científica, tecnológica e humanística.

Descritores: Educação em Odontologia; Ensino; Currículo.

\section{INTRODUÇÃO}

A partir da década de 90 , com a regulamentação da Constituição Federal de 1988 e a publicação da Lei 8080/90, as discussões sobre a formação profissional em saúde no país se intensificaram amplamente ${ }^{1,2}$. Neste cenário, entre os anos de 1998 e 1999, diversas reuniões aconteceram com a finalidade de subsidiar a implantação das Diretrizes Curriculares Nacionais (DCNs) do curso de Odontologia no Brasil, a qual ocorreu a partir do ano de 2002 por meio do Parecer CES/CNE 1.300/2001, de 06 de novembro de 2001 e a Resolução CNE/CES 3, de 19 de fevereiro 2002, publicada no Diário Oficial da União em 4 de março de 2002. A partir da publicação do Diário Oficial em 2002 iniciaram-se 
tomadas de providências para que as Instituições de Ensino Superior (IES) dessem seus primeiros passos para implementação das DCNs do curso de Odontologia ${ }^{3,4,5}$.

Diante disso, esse tema passou a ser foco de diversos encontros e reuniões embasando a realização de Oficinas de Trabalho em cursos de Odontologia, com intuito de realizar reflexões, auto-avaliações e a identificação de nós críticos para que pudessemser estabelecidos planos de ações com vistas à implantação das $\mathrm{DCNs}^{3}$.

As DCNs de Odontologia definem os objetivosdo cursoassim como os princípios, fundamentos, condições e procedimentos da formação de cirurgiões-dentistas. Essas diretrizes devem ter aplicação nacional e serem utilizadas como eixo orientador na elaboração dos currículos implementados por todas as instituições de ensino superior, sendo complementadaspor uma porção diversificada, que reflita a experiência de cada escola e as necessidades e imposições da região na qual se situa ${ }^{6}$.

A formação em Odontologia sempre esteve pautada prioritariamente no exercício privado da profissão, com ênfase no biologicismo, na sofísticação de técnicas e instrumentos. No entanto, políticas públicas em saúde, como a inserção da saúde bucal na "Estratégia de Saúde da Família" e o anúncio da prioridade dada pelo governo federal ao programa "Brasil Sorridente" fizeram com que os serviços públicos passassem a compor um significativo mercado de trabalho para os profissionais da Odontologia. Estas propostas, entretanto, defrontam-se com a precária disponibilidade de profissionais de saúde dotados de visão humanística e preparados para prestar cuidados contínuos e resolutivos à comunidade, funcionando como a porta de entrada do sistema de saúde. Para superação desse obstáculo, ocorreram mudanças no ensino tra- dicional nas instituições acadêmicas, associadas ao cumprimento das DCNs, uma vez que as políticas do Ministério da Saúde, relatam a necessidade de diminuição do distanciamento entre a formação dos profissionais de saúde e as necessidades do Sistema Único de saúde (SUS) ${ }^{3,7}$.

Seguindo as conjunturas previstas, o curso de Odontologiada Universidade Estadual de Londrina (UEL) buscou atingir o patamar exigido por meio de uma matriz curricular organizada em módulos que proporcionam maior integração entre os conteúdos, melhor racionalização do tempo, favorecendo a inserção de temas atuais e importantes para a formação profissional ${ }^{2}$. Os módulos têm por objetivo possibilitar aos alunos o trabalho em equipes multiprofissionais de saúde, além de capacitá-lo para atuar em serviços públicos de saúde em diferentes níveis de complexidade, buscando reduzir o distanciamento entre a escola e o modo de prestação real dos serviços, pautado na excelência técnica e em evidências científicas ${ }^{8}$.

Além disso, com a finalidade de fortaleceras ações multiprofissionais e intersetoriais direcionadas à atenção básica e à vigilância em saúde, de acordo com os princípios e necessidades do Sistema Único de Saúde - SUS, o curso de Odontologia da UEL também aderiu ao Programa de Educação pelo Trabalho pela Saúde(PETSaúde). Este programa encontra-se embasado no pressuposto da integração ensinoserviço-comunidade, sendo uma parceria entre a Secretaria de Gestão do Trabalho e da Educação na Saúde(SGTES), Secretaria de Atenção à Saúde (SAS) e Secretaria de Vigilância em Saúde(SVS), do Ministério da Saúdee a Secretaria de Educação Superior(SESU), do Ministério da Educação e uma importante estratégia do Programa 
Nacional de Reorientação da Formação Profissional em Saúde, o Pró-Saúde ${ }^{6,9}$.

O presente trabalho tem como objetivo relatar a experiência de estudantes do curso de Odontologia da UEL que vivenciaram o Programa PET-Saúde como facilitador em sua formação profissional,refletindo sobre os aspectos potencializadores e dificultadores, tendo em vista a formação profissional da área da saúde e o cumprimento das regulamentações propostas pelas DCNs por meio da matriz curricular do curso.

\section{MATERIAIS E MÉTODOS}

Trata-se de um trabalho retrospectivo, descritivo que discute como os autores do presente estudo, que vivenciaram ativamente o PET-Saúde daUEL,obtiveram aprendizagem significativa na perspectiva da multiprofissionalidade e intersetorialidade.

Um estudo exploratório foi realizado por meio de um levantamento bibliográfico em bases eletrônicas de dados (BBO, LILACS e SciELO) utilizando os descritores "Educação em Odontologia", "Ensino" e "Currículo" com a finalidade de se obter uma fundamentação teórica sobre a temática.O relato foi organizado em tópicos referentes àscompetências profissionais exigidas pelas DCNs do curso de Odontologia que foram desenvolvidas durante a experiência.

\section{RELATO DE EXPERIÊNCIA}

As DCNs do curso de Odontologia preconizam competências profissionaisque os estudantes do curso devem desenvolver a partir da organização da matriz curricular e da articulação com programas como o PETSaúde. A seguir algumasdessas competências serão descritas de acordo com as experiências vivenciadas por alguns estudantes do curso de Odontologia da UEL participantes do PET-Saúde.

\subsection{Coleta, observação e interpretação de dados para a construção do diagnós- tico}

O programa PET-Saúde juntamente com as disciplinas da matriz curricular do curso de Odontologia da UEL permitem que os estudantes obtenham maior contato com os serviços públicos de saúde, participando ativamente da coleta de dados por meio de observação e intervenção, e da interpretação dos mesmos para a construção do diagnóstico, quer seja diagnóstico sócio-econômico-cultural, quer seja clínico, em uma equipe multiprofissional.

Assim,as atividades de territorialização, acolhimento, visitas domiciliares, criação de vínculo eacompanhamento de atendimentos à população executadas pelos estudantes nos estágios extra-muros contribuem para o desenvolvimentos dessas competências.

\subsection{Realização da promoção e manuten- ção da saúde}

Visando uma maior integração entre os serviços de saúde e a comunidade e uma maior qualificação da atenção à população, os estudantes de Odontologia da UEL, integrantes do PET-Saúde, são estimulados a desenvolver atividades de promoção e prevenção da saúde por meio de reuniões com equipes multiprofissionais, desenvolvimento de materiais, palestras educativas e criação de grupos de atividades para a população. Além disso, levantamentos epidemiológicos são desenvolvidos, auxiliando a melhor compreensão do local e de suas necessidadese embasando discussões eestudos de $\operatorname{casos}^{10}$. 
4 TRABALHO EM EQUIPES INTERDISCIPLINARES E MULTIPROFISSIONAIS ATUANDO COMO AGENTE DE PROMOÇÃO DE SAÚDE E O DESENVOLVIMENTO DE RACIOCÍNIO LÓGICO E ANÁLISE CRÍTICA NAS DIVERSAS CONDUTAS CLÍNICAS

Tanto o projeto Pet-Saúde quanto as disciplinas da matriz curricular do curso de Odontologia da UEL permitem uma participação ativa dos estudantes em trabalhos e atividades de caráter interdisciplinar e multiprofissional $^{8}$.OPET-Saúde, por sua vez, como um programa voltado para os cursos de Educação Física, Enfermagem, Farmácia, Fisioterapia, Medicina, Odontologia e Serviço Social auxilia no desenvolvimento dessas ações em saúde, uma vez que grupos multiprofissionais com estudantes de distintas áreas são formados estimulando o trabalho multiprofissional, o desenvolvimento de raciocínio lógico, a análise crítica em diversas condutas, as tomadas de decisões e o trabalho em equipe ${ }^{11}$.

Assim, em um trabalho multiprofissional uma troca expressiva de experiências ocorre sobre a ótica de uma abordagem integral e resolutiva, o que viabiliza o planejamento de ações de saúde mais eficazes, o que ocorre por meio do PET-Saúde, onde a prestação da atenção integral às famílias com ações educativas, preventivas e clínicas tornam-se o foco da atenção ${ }^{10,12}$.

\section{PLANEJAMENTO E ADMINIS- TRAÇÃO DOS SERVIÇOS DE SA- ÚDE COLETIVA}

Durante o desenvolvimento do PET-

Saúde e das atividades propostas pelas disciplinas da matriz curricular do curso de Odontologia da UEL, os estudantes são estimulados a planejar ações a fim de proporcionar melhorias na qualidade de vida e saúde da população. São desenvolvidas atividades práticas de gestão dos serviços como a organização da demandaa partir de grupos prioritários nas Unidades Básicas de Saúde, dos municípios de Londrina e região, de modo a proporcionar a universalidade, integralidade e equidade do atendimento.

Assim, a partir da observaçãoda estrutura e funcionamento das Unidades Básicas de Saúde (UBS) os estudantestêm a oportunidade de conhecerin loco asdistintas propostas de cada UBS assim como o planejamento local, o funcionamento do controle social e os programas desenvolvidos para a comunidade ${ }^{10}$.

\section{DISCUSSÃO}

O PET-Saúde se insere como uma estratégia do Pró-Saúde, que incentiva a formação de grupos de aprendizagem tutorial por meio da iniciação ao trabalho multiprofissional e interdisciplinar dos estudantes dos cursos de graduação na área da saúde, constituindo-se como uma iniciativa intersetorial direcionada ao fortalecimento da integração ensino-serviço no âmbito da atenção básica. Iniciativas como as do PETSaúde buscam atender o que é preconizado tanto pelas diretrizes curriculares nacionais para a formação dos profissionais da saúde, quanto pelas diretrizes para o exercício profissional no $\mathrm{SUS}^{12}$.

A Lei Orgânica 8080/90, artigo 27 reconhece que os serviços públicos que integram o SUS constituem um campo de práticas para o ensino e a pesquisa, de modo a articular os interesses das Instituições de Educação Superior (IES) e do SUS, com vistas à melhoria da qualidade do atendimento à população ${ }^{1,2}$.

As DCNs dos Cursos de Graduação na área da saúde, definidas para todos os 
cursos desta área, apontam que "a formação do profissional deve contemplar o sistema de saúde vigente no país, o Sistema Único de Saúde - SUS, baseado na atenção integral da saúde em um sistema regionalizado e hierarquizado de referência e contrarreferência e no trabalho em equipes" .

A Resolução CNE/ CES n³, de 19 de fevereiro de 2002 instituiu as Diretrizes Curriculares Nacionais para o Curso de Graduação em Odontologia, objetivando a formação geral e específica dos egressos/profissionais com ênfase na promoção, prevenção,recuperação e reabilitação da saúde" 5 .

O Cirurgião-Dentista graduado de acordo com essa resolução deve apresentar uma formação generalista, humanista, críticae reflexiva para atuar em todos os níveis de atenção à saúde, com base no rigor técnico e científico, sendo capacitado ao exercício de atividades referentes à saúde bucal da população, pautado em princípios éticos, legais e na compreensão da realidade social,cultural e econômica do seu meio, dirigindo sua atuação para a transformação da realidade em benefício da sociedade ${ }^{7}$.
Dentro desses preceitos, e com intuito de acatar as proposições das DCNs, a Universidade Estadual de Londrina (UEL) passou por uma reforma em sua matriz curricular no ano de 2005, e a partir de então o currículo passou a ser organizado em módulos integrados. Dentre esses módulos, dois merecem destaque, o primeiro deles alocado na $4^{\mathrm{a}}$ série do curso, intitulado"Estágio Supervisionado em Serviços de Saúde dos Municípios da Região-Urbana e Rural", e o segundo alocado na $5^{\text {a }}$ série do curso denominado,"Estágio Supervisionado em Equipe Multiprofissional", tendo em vista que ambos se desenvolvem em consonância com o PET-Saúdeda UEL (Figura $1)$.

Essas disciplinas proporcionam aos estudantes de odontologia a possibilidade de atuarem em equipes multiprofissionais e interdisciplinares da rede pública de saúde, capacitando o graduando para o planejamento de serviços, realização de educação comunitária, vivência das praticas de gestão, controle e avaliação dos serviços de saúde, além da realização de procedimentos educativos, preventivos e curativos nos diferentes níveis de complexidade ${ }^{8}$. 
Figura 1 - Interrelação dos módulos integrados da matriz curricular do curso de Odontologia da UEL e o PET-Saúde no desenvolvimento competências profissionais preconizadas pelas DCNs

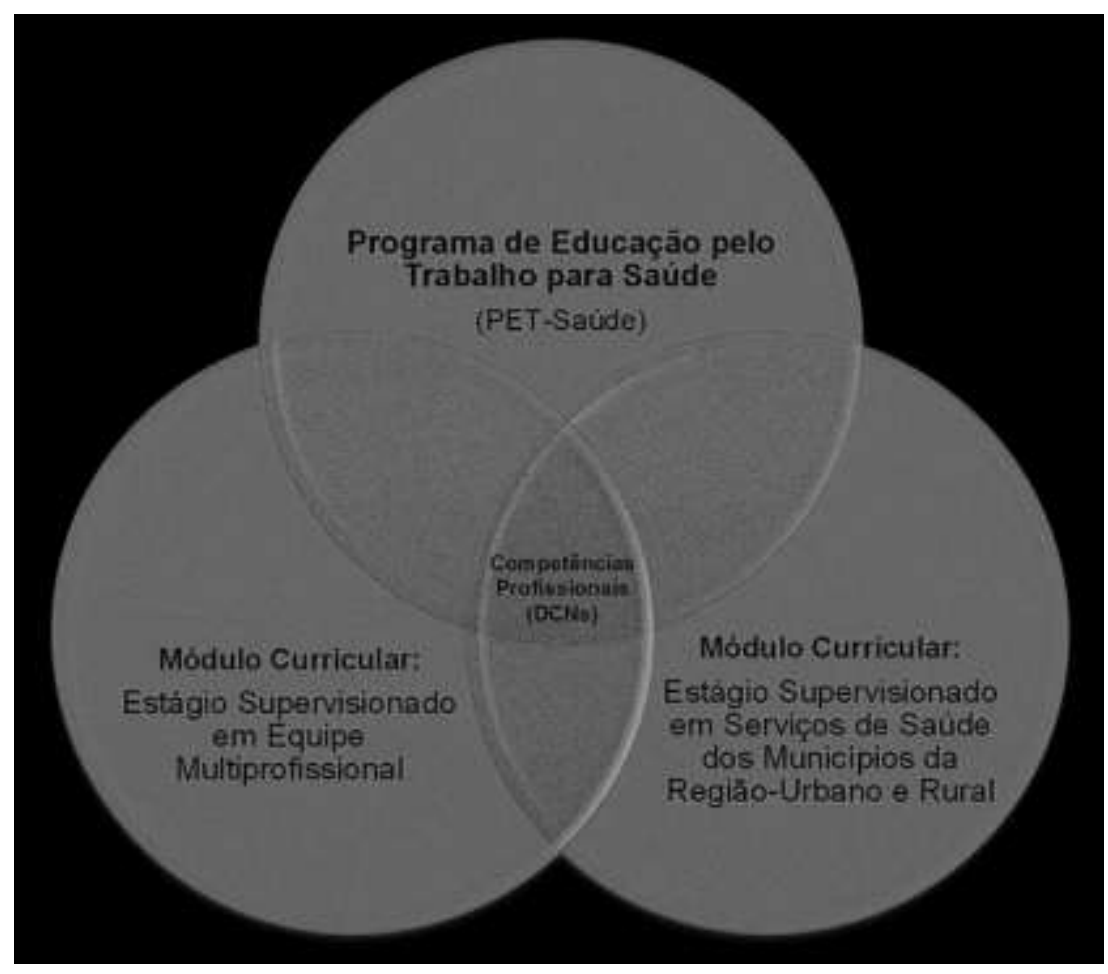

\section{CONCLUSÃO}

É perceptível que tanto as atividades desenvolvidas pelo PET-Saúde quanto às preconizadas pela matriz curricular do curso de Odontologia da UEL priorizam formar profissionais mais decisivos e desenvoltos, com conhecimentos e atividades que os tornem competentes para atuar no SUS, com amadurecimento profissional e elevada formação científica, tecnológica e humanística, tornando-se uma excelente proposta de integração entre o trabalho em saúde e a educação, incentivando a articulação entre o ensino, a pesquisa e a extensão na área de saúde coletiva, com foco na Atenção Básica.

Logo, as atividades desenvolvidas pelos alunos graduandos em Odonto logia pela UEL e participantes do PETSaúde encontram-se em consonância com os pressupostos das DCNs, apontando significativa relevância desse projeto para a formação profissional associadas às necessidades da população. Além disso, o PETSaúde vem favorecendo a integração ensino-serviço que, além de preconizada pelas DCNs, é fundamental para o desenvolvimento das competências requeridas pelas mesmas.

\section{REFERÊNCIAS}

1. Cavalheiro MTP, Guimaraes AL. Formação para o SUS e os Desafios da Integração Ensino Serviço. Caderno FNEPAS 2011; 1(1): 1-9. 
2. Brasil. Lei $\mathrm{n}^{\mathrm{o}} 8080$, de 19 de setembro de 1990. Dispõe sobre as condições para a promoção, proteção e recuperação da saúde, a organização e o funcionamento dos serviços correspondentes e dá outras providências. Diário Oficial da União. Brasília, 20 set. 1990; Seção 1.

3. Morita MC, Kriger L, Carvalho ACP, Haddad AD. Implantação das Diretrizes Curriculares Nacionais em Odontologia [Internet]. Maringá: Editora Dental Press, 2007 [citado em 02/02/2014].Disponível em: http://abeno.org.br/arquivos/downloads /download_20111109153352.pdf

4. Brasil. CNE. Parecer CES/CNE 1300/2001 [internet]. Diário Oficial da União, Seção 1, p. 25; Brasília, 7 de dezembro de 2001- [acesso em 02/02/2014]. Disponível em: http://portal.mec.gov.br/cne/arquivos/p df/CES1300.pdf

5. Brasil. CNE. Resolução CNE/CES 3/2002 [internet]. Diário Oficial da União, Seção 1, p.10; Brasília, 4 de março de 2002 - [acesso em 10/12/2013]. Disponível em: http://portal.mec.gov.br/cne/arquivos/p df/CES032002.pdf

6. Carvalho ACP. Planejamento do curso de graduação de Odontologia: É importante planejar os cursos de graduação considerando-se as Diretrizes Curriculares Nacionais. Revista da Abeno 2004; 4 (1): 7-13.

7. Carvalho BR, Costa TBC, Gomes MJ, Santos KT, Guerra SMG. Formação docente em Odontologia no Brasil: sugestões de mudanças após as Diretrizes Curriculares Nacionais. Revista Brasileira de Pesquisa em Saúde 2010; 12(4): 39-44.
8. Curso de Odontologia da Universidade Estadual de Londrina [internet]. Página da Universidade Estadual de Londrina (BR); 2010- [acesso em 08/12/2013]. Disponível

em:http://www.uel.br/graduacao/odont olo-

gia/index.php?option=com_content\&vi $\mathrm{ew}=$ article $\& \mathrm{id}=74 \&$ Itemid $=11$

9. Projeto Pró-Saúde. Curso de Odontologia da Universidade Estadual de Londrina [internet]. Página da Universidade Estadual de Londrina (BR); 2010[acesso em 08/12/2013]. Disponível em:http:/www.uel.br/ccs/odontologia/i ndex.php/pro-saude.

10. Buffon MCM, Carvalho DS, Daniel E, Junior HS, Pecharki GD, Mariot CA, Ferreira CM, Santos GBS, MadureiraJunior JS, Massaro DCC, Mosimann MC, Bergami RH, Geraldo LC, Kai LKK. Contribuição do PET-Saúde para a área de odontologia da UFPR na consolidação das Diretrizes Curriculares Nacionais e do SUS, nos municípios de Curitiba e Colombo-PR. Revista da Abeno 2011; 11(1): 9-15.

11. Ministério da Saúde [Internet]. Página do Ministério da Saúde (BR) 2013 [acesso em 10/12/2013]. Disponível em:

http://portal.saude.gov.br/portal/sau de/profissional/visualizar_texto.cfm?idt $\mathrm{xt}=35306$

12. Sobrinho TAO, Medeiros CPP, Maia MR, Reis TC, Miranda LP, Costa PF.Integração Acadêmica e Multiprofissional no Pet-Saúde: Experiências e Desafios. Revista da Abeno 2011; 11(1): 39-42. 


\section{ABSTRACT}

The PET-Health in consonance with the National Curriculum Guidelines of Dentistry in the development of professional competencies - Experience Report

The National Curriculum Guidelines of Dentistry (DCNs) define the course objectives and principles, fundamentals, conditions and procedures of the training of dentists and must have national application being used as a guiding principle in the development of curriculum implemented in higher education institutions. The State University of Londrina (UEL) looking for reach the level required by DCNs based their curriculum in integrated modules and in line with them adhered the Educational Program for Health Work (PET-Health). This study aimed to report the experiences of the authors of this article, who experi- enced the PET-Health of UEL as facilitator in their professional formations. The report was organized into topics about professional competencies required by DCNs of Dentistry that were developed during the experience. It was concluded that both the activities of the PET-Health as those recommended by the curriculum of the Dentistry course of UEL prioritize form more decisive and resourceful professionals with knowledge and activities that make them competent to act in the Health System (SUS), with professional ripening and high scientific, technological and humanistic formation.

Descriptors: Education, Dental; Teaching; Curriculum. 